\title{
Uso de hidrolizados de pescado en la acuicultura: una revisión de algunos resultados beneficiosos en dietas acuícolas
}

\author{
Use of fish hydrolysate in aquaculture: a review of \\ some beneficial results in aquafeeds
}

\author{
Alessandra L. Cardoza Ramirez; Mariafernanda G. Guerra Espinoza1; Alfredo R. Palomino Ramos ${ }^{1, *}$ \\ 1 Departamento de Biología Marina e Ingeniería Acuícola, Universidad Científica del Sur, Carretera Panamericana Sur Km. \\ 18, Lima, Perú.
}

*Autor corresponsal: apalominor@cientifica.edu.pe (A. R. Palomino Ramos).

ID ORCID de los autores

A. L. Cardoza Ramirez: iD http://orcid.org/0000-0002-5031-955X
M. G. Guerra Espinoza: iD http://orcid.org/0000-0002-4103-0180
A. R. Palomino Ramos: iD http://orcid.org/0000-0003-0510-3124

\section{RESUMEN}

Las industrias pesqueras y de acuicultura generan, cada año, un conjunto de residuos o desechos que incluyen piel, cabeza, vísceras, recortes y espinazos, representando más del $60 \%$ en volumen productivo. Estos residuos tienen altos contenidos de proteínas, y normalmente son procesados en productos de bajo valor comercial, como alimentos para animales, harina de residuos y fertilizantes. En los últimos años, se han venido desarrollando tecnologías para el aprovechamiento de éstos residuos y convertirlos en bioproductos de mayor valor agregado, como son los hidrolizados de proteínas, con interesantes aplicaciones en la alimentación animal. Los hidrolizados proteicos de pescado son productos obtenidos de la degradación enzimática o química de las proteínas de pescado en péptidos más pequeños, aminoácidos libres y nucleótidos, obteniéndose un alto contenido proteico con buen balance de aminoácidos, alta digestibilidad y mejor aprovechamiento de sus nutrientes. La inclusión de éstos hidrolizados en los alimentos acuícolas puede mejorar el crecimiento y la eficiencia alimentaria de los organismos acuáticos en cultivo. Este artículo presenta una revisión sobre investigaciones de la inclusión de hidrolizados proteicos de pescado en dietas experimentales para peces, crustáceos, moluscos y algas, y los efectos en los desempeños productivos, en los últimos veinte años.

Palabras clave: organismos acuáticos; desempeño productivo; eficiencia alimentaria; tasa de crecimiento.

\section{ABSTRACT}

Each year, the fishing and aquaculture industries generates a set of residues or wastes including skins, heads, viscera, trimmings and bones, representing more than $60 \%$ of the productive volume. These residues are high in protein, and are normally processed into products of low commercial value, such as animal feed, fish meal and fertilizers. In recent years, diverse technologies have been developed to make use of these residues and convert them into useful bioproducts of higher value, such as protein hydrolysates, with interesting applications in animal feed. Fish protein hydrolysates are products obtained from the enzymatic or chemical degradation of fish proteins into smaller peptides, free amino acids and nucleotides, obtaining high protein contents with good amino acid balance, high digestibility and more efficient use of its nutrients. The inclusion of these hydrolysates in aquafeeds can improve the growth rate and feed efficiency of cultured aquatic organisms. This article presents a review on research of the inclusion of fish protein hydrolysates in experimental diets for fish, crustaceans, mollusks and algae, and the effects on its production performances, in the last twenty years.

Keywords: aquatic organisms; productive performance; feed efficiency; growth rates

Recibido: 06-03-2021.

Aceptado: 21-04-2021.

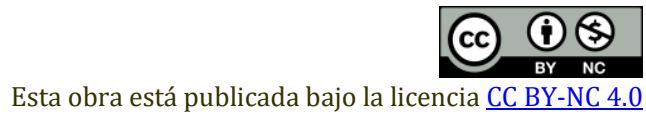




\section{INTRODUCCIÓN}

La importancia de la industria pesquera radica en su enorme participación en el comercio mundial de alimentos, con aproximadamente 179 millones de toneladas de producción, incluida la acuicultura, en la que 156 millones fueron para consumo humano (FAO, 2020). Sin embargo, esta industria actualmente genera residuos ricos en proteínas, los cuales incluyen cabeza, piel, escamas, aletas, recortes, vísceras (principalmente estómago, ciegos pilóricos e intestino, como fuente importante de proteasas) y hueveras, siendo descartados sin intentos de recuperación y que representan, dependiendo de la especie, entre 50 al 70\% (Silva et al., 2014; Villamil et al., 2017).

Estas materias subutilizadas podrían constituirse en un aditivo o ingrediente funcional para la alimentación animal, o como alternativa a la harina de pescado, en la estrategia de su sustitución o reemplazo por ingredientes no tradicionales (Refstie et al., 2004; Xu et al., 2016). El hidrolizado de proteína de pescado (HPP o FPH, por su sigla en inglés) producido a partir de estos residuos, tiene un alto contenido de proteínas, un buen equilibrio de aminoácidos y ácidos grasos, una alta digestibilidad y atractabilidad, excelente textura y viscosidad, y un tamaño de partícula muy pequeño, que mejoran su funcionalidad $\mathrm{y}$ facilitan la absorción de nutrientes (Chotikachinda et al., 2013; Ospina-Salazar et al., 2016; Valle et al., 2015).
Los hidrolizados presentan propiedades nutricionales y funcionales capaces de aumentar el desempeño productivo de los organismos al ser suplementados en las dietas, de modo que favorecen la nutrición básica y la mejora de la salud, pudiendo ser apropiados para la alimentación acuícola, como ha sido demostrado en varias especies de cultivo, incluyendo peces, crustáceos y algunos moluscos (Galla et al., 2012; Xu et al., 2016).

Como es sabido, la alimentación es un punto crucial en la actividad acuícola, al representar en muchos casos, más del $60 \%$ de los costos de producción (Han et al., 2018), siendo necesario para los centros acuícolas, establecer ciertas estrategias que les permitan reducir los costos, y a la vez, mejorar la eficiencia alimenticia, por lo que una de esas estrategias sería la inclusión de ingredientes funcionales, como es el caso de los HPP.

La importancia del presente artículo de revisión radica en el aporte informativo relevante de los beneficios de incluir HPP en las dietas acuícolas y la mejora sobre el desempeño productivo de peces $u$ otros invertebrados de cultivo, además de fomentar su uso como ingrediente funcional, el cual es obtenido como parte de los subproductos generados por la industria pesquera y acuícola, reduciéndose de esta forma el impacto sobre el ambiente.

\section{MATERIAL Y MÉTODOS}

El presente trabajo se efectuó mediante una investigación sistemática de artículos científicos y otros documentos técnicos publicados en inglés, portugués y en español, que figuran en las bases de datos de Scielo, Sciencedirect, Scopus, Springer Link y Wiley Online Library, durante el periodo 2001 2021.

Respecto al procesamiento de la información, se emplearon criterios de inclusión y exclusión. En primer lugar, se consideraron todos los documentos o artículos relacionados a la incorporación de los hidrolizados de pescado en dietas para organismos acuáticos. Los principales criterios de exclusión se refirieron a aquellos que no presentaron una relación marcada con nuestro objetivo, es decir, no consideraban los efectos de los hidrolizados sobre el desempeño productivo de las especies en estudio.

Los documentos considerados como incluidos fueron organizados en cinco categorías: caracterización de los hidrolizados de pescado, y los efectos benéficos en peces, crustáceos, moluscos y algas. Después de categorizarlos, se procedió a la revisión de la información mediante técnicas comparativas y analíticas, con el propósito de fomentar la discusión y plasmar la información relevante y novedosa para una revisión científica según los objetivos planteados.

\section{RESULTADOS Y DISCUSIÓN}

Hidrolizado proteico de pescado 1. Definición de hidrolizado proteico

Los hidrolizados de proteína de pescado (HPP) son bioproductos obtenidos del aprovechamiento eficiente de los residuos generados de la industria pesquera y/o acuícola, permitiendo la solubilización de la fuente de proteína para mejorar su valor biológico y nutricional (Chalamaiah et al., 2012; He et al., 2013; Quinto et al., 2018), siendo su presentación final en polvo y de coloración blanco cremoso, aunque algunas veces puede ser en forma líquida (He et al., 2013; Villamil et al., 2017).

Bioquímicamente, la hidrólisis de proteínas consiste en la escisión de enlaces peptídicos para obtener aminoácidos libres y péptidos de bajo peso molecular (conteniendo entre 2 - 20 aminoácidos) y cada enlace roto consume una molécula de agua (Halim et al., 2016; Villamil et al., 2017). Como resultado de este proceso, presentan mayor facilidad de absorción por los enterocitos en comparación con las macromoléculas de alto peso molecular, haciéndolos altamente digestibles (Mamauag et al., 2016; Quinto et al., 2018).

\section{Materia prima de HPP}

Las materias primas para los HPP provienen de los residuos del procesamiento pesquero, y éstas pueden provenir de tres componentes principales del pescado. 
Músculo: Está referido principalmente a la parte oscura del pescado, rico en proteínas estructurales, miofibrilares y sarcoplasmáticas, que contienen todos los aminoácidos esenciales, entre los que predominan lisina, fenilalanina y valina. No obstante, tiene un valor limitado debido a su oxidación potencial, pudiendo causar inconvenientes en el consumidor, por lo que presenta un bajo valor de mercado (Chalamaiah et al, 2012; Halim et al., 2016). Representa entre el 15 - 20\% de la materia prima cruda (Refstie et al., 2004).

Piel y aletas: Es un subproducto del procesamiento que generalmente se elimina y es fuente rica en colágeno y gelatina (Chalamaiah et al., 2012; Halim et al., 2016). Representa entre el 1\% y 3\% de la materia prima cruda (Villamil et al., 2017).

Desperdicios: Subproductos que incluyen cabeza $(9 \%-12 \%)$, espinazos y huesos $(9 \%-15 \%)$, restos de carcasa, escamas (5\%), vísceras y gónadas (12\% - 18\%) (Halim et al., 2016; Villamil et al., 2017).

\section{Métodos de hidrolizados}

Las proteínas pueden hidrolizarse mediante procesos químicos, reacciones enzimáticas, autolisis, hidrólisis térmica y fermentación bacteriana, obteniéndose diferentes calidades (Halim et al., 2016; Wisuthiphaet y Kongruang, 2015). La producción de un hidrolizado puede ser dividida en tres procesos: pre-tratamiento, hidrolización y recuperación (He et al., 2013).

En el primer proceso, la materia prima se mezcla con el agua en proporciones iguales para formar una suspensión homogénea (He et al., 2013). Esta suspensión es hidrolizada según las condiciones propias del método empleado, como temperatura, tiempo de hidrólisis, $\mathrm{pH}$ o grado de hidrólisis (Costa et al., 2020). En la etapa de recuperación, el centrifugado separa la suspensión hidrolizada en tres capas: una capa de aceite (parte superior), una solución de hidrolizado de proteínas (parte media) y una capa semisólida (parte inferior) (Siddick et al., 2021). Luego, la parte media es retirada y secada por aspersión o liofilizada, dependiendo del nivel de producción (Siddick et al., 2021).

En la producción de HPP, la hidrólisis química y enzimática son los métodos más comunes de producción, sin embargo, los investigadores mencionan que suelen utilizar la hidrólisis enzimática, porque es rápida y controlable, además de conservar el valor nutricional del producto (Halim et al., 2016; Wisuthiphaet y Kongruang, 2015). La caracterización del producto final viene dada por el grado de hidrólisis (GH), definido como el porcentaje de enlaces peptídicos rotos en relación con la proteína original (Zamora-Sillero et al., 2018).

\subsection{Método químico}

Este método se realiza mediante una hidrólisis ácida o alcalina, y se considera un método convencional para producir HPP. Se utilizan ácidos fuertes o compuestos alcalinos para escindir enlaces peptídicos a alta presión $(100 \mathrm{kPa}) \mathrm{y}$ temperatura $\left(120{ }^{\circ} \mathrm{C}\right)$. Este método demanda menos costo y tiempo, sin embargo, la reacción de hidrólisis es de difícil control, debido a la fuerte reacción química y la escisión de enlaces peptídicos inespecíficos, afectando la calidad del producto (He et al., 2013; Wisuthiphaet \& Kongruang, 2015).

Como consecuencia, este proceso puede provocar la conversión de L-aminoácidos en D-aminoácidos, que no pueden ser utilizados por humanos ni animales (Wisuthiphaet y Kongruang, 2015). Algunos aminoácidos esenciales como triptófano, metionina y cisteína disminuyen durante la reacción o son destruídos (Villamil et al., 2017; Wisuthiphaet \& Kongruang, 2015). Además, durante la reacción de hidrólisis, los residuos hidrófobos generados originan un amargor del HPP (Wisuthiphaet \& Kongruang, 2015), limitando el uso de los mismos en la alimentación animal. El HPP producido se suele utilizar como aditivo en dietas balanceadas, medios de cultivo $y$ fertilizantes para plantas (Villamil et al., 2017).

\subsection{Método biológico}

Este método se basa en el uso de enzimas endógenas o exógenas, del tipo peptídicas denominadas proteasas, proteinasas, peptidasas o enzimas proteolíticas, las cuales hidrolizan los enlaces peptídicos (Chalamaiah et al., 2012; Halim et al., 2016). Esta hidrólisis es más ventajosa que el método químico, porque permite un mayor control y selectividad del proceso, siendo menos drástico y generando un producto de mayor valor nutricional (Zamora-Sillero, 2018). El perfil peptídico del producto final está afectado por factores como: concentración de sustrato proteico, tipo de enzima, concentración de enzima $(0,01 \%-5,0 \%), \mathrm{pH}(1,5-$ $11,0)$, temperatura $\left(35-60^{\circ} \mathrm{C}\right)$ y tiempo $(10-600$ min), los cuales influyen en gran medida sobre la reacción cinética de las enzimas (Siddik et al., 2021). Generalmente, existe una combinación óptima de $\mathrm{pH}$ y temperatura en la que una enzima es más activa, y también una combinación en la que una enzima puede desactivarse mediante la desnaturalización (Zamora-Sillero, 2018).

\section{Composición proximal}

Los HPP se caracterizan por presentar altos tenores proteicos $(60 \%-90 \%)$ y niveles de lípidos por debajo del 5\% (en la mayoría de los casos) (Chalamaiah et al., 2012; Siddik et al., 2021). El contenido de proteína es mayor debido a la solubilización de las mismas, producto del retiro de material no proteico insoluble y eliminación de grasa (Mamauag et al., 2016). Esta última explicaría el bajo contenido de grasas (Chalamaiah et al., 2012), dando estabilidad a los HPP frente a la oxidación de lípidos, aumentando la vida útil en condiciones de almacenamiento (Siddik et al., 2021). En cuanto a la humedad, la mayoría de los estudios reportaron niveles inferiores al 10\%, dependiendo de la materia prima utilizada y de las temperaturas altas empleadas durante el proceso de evaporación y secado (Chalamaiah et al., 2012).

\section{Perfil de aminoácidos y fracciones proteicas} Los HPP presentan variaciones en su composición de aminoácidos y depende principalmente de varios factores, como la materia prima, la fuente de 
enzimas y las condiciones de hidrólisis (Klompong et al., 2009). Diversos estudios han reportado que los HPP (principalmente a partir de músculo, cabeza, piel y vísceras) contienen todos los aminoácidos esenciales y no esenciales (Siddik et al., 2021). Entre los aminoácidos, el ácido aspártico y el ácido glutámico presentan los niveles más altos en la mayoría de los HPP reportados (Chalamaiah et al., 2012).

En relación al grado de hidrólisis, cuanto mayor es el valor, se tendrá una mayor ruptura de enlaces peptídicos, produciendo más cantidad de péptidos en la solución, aumentando la solubilidad de la proteína y su posibilidad de recuperación para ser utilizada como aditivo alimenticio (Sheriff et al., 2014). De acuerdo a los diversos estudios, el grado de hidrólisis es variable para permitir obtener el tamaño de las fracciones proteicas apropiadas para el consumo animal, obteniéndose valores por encima de $35 \%$ y hasta debajo de $50 \%$.

El HPP contiene compuestos de diferente peso moleculares que oscilan de decenas a miles de Da, los cuales pueden afectar la capacidad de absorción y la velocidad de paso de los nutrientes a través del tracto gastrointestinal (Cai et al., 2015). Generalmente, los pesos moleculares de los péptidos de los HPP están por debajo de 3000 a 5000 Da (Bui et al., 2014), donde las fracciones proteicas se pueden distribuir de la siguiente forma: < $200 \mathrm{Da}$ (aminoácidos libres), 200 - $500 \mathrm{Da}$ (di/tripéptidos), 500 - 2500 Da (oligopéptidos con más de tres residuos), y > 2500 Da (proteínas/ polipéptidos). Algunos estudios han establecido que los HPP deberían contener una mayor proporción de péptidos en el rango de 500 - 2500 $\mathrm{Da}$, seguido de péptidos entre 200 - $500 \mathrm{Da}$ (Kotzamanis et al., 2007). Asimismo, estudios en alimentación con peces y crustáceos sugieren que los dipéptidos y tripéptidos tienen una mejor absorción que los aminoácidos libres (Quinto et al., 2018).

\section{Uso en la alimentación acuícola 6.1 Efectos sobre el desempeño productivo de peces}

El uso de hidrolizados como ingredientes en alimentos balanceados de peces, puede mejorar su desempeño productivo, como el crecimiento, la ganancia de peso, la eficiencia alimentaria y la reducción de la mortalidad.

Este efecto promotor del crecimiento se puede atribuir, en primer lugar, a las propiedades de atractabilidad o aromatizantes de los HPP sobre los alimentos balanceados, explicado por la presencia de aminoácidos libres (ácido glutámico, ácido aspártico, glicina, arginina, alanina, prolina, leucina e isoleucina) y algunos nucleótidos (Chotikachinda et al., 2013; Quinto et al., 2018). El sistema gustativo de los peces es sensible a estos compuestos solubles y diluidos en el agua (Ha et al., 2019). Los resultados de ésta inclusión han promovido un mayor consumo de alimento en lubina asiática (Lates calcarifer) (Chotikachinda et al., 2013); juveniles de salmón Chinook (Oncorhynchus tshawytscha Walbaum) (Ho et al., 2014), larvas de lubina europea (Dicentrarchus labrax) (Kotzamanis et al., 2007), post-smolt de salmón del Atlántico (Salmo salar) (Refstie et al., 2004), juveniles de mero (Epinephelus fuscoguttatus) (Mamauag et al., 2016) y juveniles de bagre sudamericano (Rhamdia quelen) (Ha et al., 2019).

La suplementación de quimioatractantes y estimulantes en dietas a base de proteínas vegetales, constituyen una estrategia alimentaria para mejorar la aceptabilidad y la ingesta de alimentos principalmente en peces marinos y carnívoros (Chotikachinda et al., 2013). La inclusión de HPP mejoró el consumo del alimento en juveniles de bacalao del Atlántico (Gadus morhua) (Akness et al., 2006a), lubina europea (Costa et al., 2020), turbot (Scophthalmus maximus) (Xu et al., 2016) y trucha arco iris (Oncorhynchus mykiss) (Akness et al., 2006a).

Asimismo, dietas suplementadas con HPP presentan alta digestibilidad, facilitando la rápida absorción de péptidos y aminoácidos a través de la membrana intestinal, aumentando el crecimiento y mejorando la eficiencia alimentaria en el lenguado japonés (Paralichthys olivaceus) (Zheng et al., 2012) y en la trucha arco iris (Akness et al., 2006b). La misma forma molecular de los HPP, dada por la presencia de aminoácidos libres o péptidos pequeños en las dietas, puede ayudar a reducir la interacción entre enzimas y sustratos, particularmente de las endopeptidasas, siendo utilizados directamente por los peces (Córdova-Murueta y GarcíaCarreño, 2002), e influenciando en la asimilación de las proteínas contenidas en la dieta.

Del mismo modo, algunos estudios permiten sugerir que las moléculas contenidas en los HPP (lisina, metionina, nucleótidos, anserina y taurina) tienen la capacidad de estimular la producción de hormonas de crecimiento similares a la insulina (IGF-I e IGF-II) (Li et al., 2018; Martínez-Alvarez et al., 2015). Estudios realizados en juveniles de lenguado japonés (Akness et al., 2006a) reportaron incrementos en el rendimiento del crecimiento, los niveles plasmáticos de IGF-I y la expresión de ARNm de IGF-I, mientras que estudios en bacalao del Atlántico (Akness et al., 2006b) y del turbot (Zheng et al., 2013) alimentados con dietas con sustitución parcial y total de harina de pescado, presentaron bajo consumo de alimentos y bajos niveles plasmáticos de IGF-I, por lo que este grupo de hormonas está fuertemente influenciado con la condición nutricional de los peces (Akness et al., 2006a; Martínez-Alvarez et al., 2015).

La inclusión de HPP como sustitutos parciales de proteínas en dietas para larvas induce a cambios en la actividad de las principales proteasas digestivas y promueve una maduración intestinal más temprana, tal como se ha evidenciado en larvas de lubina europea (Kotzamanis et al., 2007) y esturión persa (Acipenser persicus) (Ovossipour et al., 2014). En post-larvas de halibut del Atlántico (Hippoglossus hippoglossus L.), la mejora de la supervivencia estuvo evidenciada por un aumento de las actividades específicas de las enzimas intestinales como aminopeptidasa $\mathrm{N}$ y fosfatasa alcalina (AP), en detrimento de la actividad de la leucina-alanina peptidasa (LAP), indicando la maduración intestinal para la digestión de nutrientes (Kvåle et al., 2009). 
En relación a la supervivencia, dietas suplementadas con HPP permitieron un incremento de ese parámetro en larvas de tilapia nilótica (Oreochromis niloticus) (Sary et al., 2017), larvas de lubina asiática (Srichanun et al., 2014) y juveniles de turbot (Wei et al., 2019). Los péptidos de bajo peso molecular presentan propiedades imnunoestimulantes y antibacterianas, activando el sistema inmunológico en los peces a través de la mejora de las respuestas inmunes no específicas. Ciertos péptidos de tamaño medio (500 - $3000 \mathrm{Da})$ son capaces de estimular la actividad de los macrófagos, así como de las lisozimas (Costa et al., 2020; Khosravi et al., 2015; Sary et al., 2017). Los HPP pueden ser una estrategia de sanidad acuícola, evitando así ocurrencia de enfermedades y el uso de antibióticos.

Los beneficios de la suplementación de HPP en dietas para peces, permite visualizar una mejora sobre los principales índices del desempeño productivo para diferentes estadios estudiados (Tabla 1). Varios estudios reportan que los beneficios de la incorporación de HPP es más evidente en las larvas de peces que en juveniles. Esto puede ser explicado por la alta demanda de aminoácidos que presentan para satisfacer los requisitos anabólicos (Cai et al., 2015). Además, en la mayoría de larvas de peces marinos, el estómago aún no se encuentra diferenciado, por lo que la tripsina tendrá la responsabilidad de la digestión de las proteínas consumidas, haciéndose crítica la disponibilidad de aminoácidos para la síntesis proteica en esa etapa (Cai et al., 2015).

\subsection{Efectos sobre el desempeño productivo de crustáceos}

Al igual que en los peces, dietas suplementadas con HPP en crustáceos han permitido una mejora en el desempeño productivo y en la supervivencia. Dentro de los crustáceos, la principal especie de cultivo es el camarón blanco Penaeus vannamei, también conocido como langostino, y es donde más trabajos se han desarrollado.
Ensayos con dietas suplementadas con HPP permitió una mejora de la atractabilidad de la dieta y estimuló la alimentación en el langostino (Grey et al., 2009). Los crustáceos son organismos quimiosensoriales primarios, por lo que los quimioatractantes y estimulantes de la alimentación (efectores de alimentación) son componentes importantes de sus presas o alimento (MartínezAlvarez et al., 2015). Ciertos aminoácidos (taurina, glicina, arginina, ácido glutámico y alanina) han sido identificados como potenciadores de la alimentación, los cuales se encuentran presentes en los HPP, y provocarían respuestas conductuales, fisiológicas y electrofisiológicas en el sistema olfativo y gustativo, permitiendo explicar los efectos positivos en el crecimiento de los crustáceos (Grey et al., 2009).

El crecimiento y la supervivencia de los camarones están estrictamente relacionados con la calidad del alimento suministrado, y particularmente, con la fuente de proteínas y un buen equilibrio de aminoácidos (Valle et al., 2015). Ejemplares de $P$. vannamei alimentados con dietas suplementadas con HPP presentaron una mejora en el peso final, ganancia de peso y tasa de crecimiento específico (Hernández et al., 2011; Quinto et al., 2013; Valle et al., 2015). Asimismo, la forma molecular de los HPP reduciría la interacción entre enzimas y sustratos (Córdova-Murueta y García-Carreño, 2002), y los crustáceos utilizarían directamente los aminoácidos para el transporte de minerales, formación y mantenimiento de tejidos, y síntesis de hormonas y enzimas (Valle et al., 2015), aumentando de esta forma la eficiencia alimentaria tal como se ha observado en langostinos (Córdova-Murueta y García-Carreño, 2002; Hernández et al., 2011; Nguyen et al., 2012). P. vannamei alimentados con altos niveles de HPP presentaron baja actividad enzimática de endopeptidadas en el hepatopáncreas, sugiriendo la capacidad de esta especie para modular su secreción enzimática de acuerdo a la proteína consumida (Córdova-Murueta y GarcíaCarreño, 2002).

Tabla 1

Efectos positivos de HPP en el cultivo de peces. Estadios: larva (l), post-larva (pl), post-smolt (ps), alevino (a), juvenil (j). Indice productivo: GP (ganancia de peso), TCE (tasa de crecimiento específico), FCA (factor de conversión alimenticia), TEP (tasa de eficiencia proteica) (Adaptado de Martínez-Alvarez et al., 2015)

\begin{tabular}{lrll}
\hline Especie & Dosis HPP & Índice afectado & Referencia \\
\hline Anguila japonesa (l) & $1.6 \%$ & Talla final & Masuda et al., 2013 \\
Bagre sudamericano (j) & $5-10 \%$ & GP, TCE, FCA & Ha et al., 2019 \\
Bagre sudamericano (j) & $25 \%$ & GP, FCA, TEP & Wosniak et al., 2016 \\
Corvina azafrán (j) & $10-15 \%$ & GP, TCE & Tang et al., 2008 \\
Esturión persa (l) & $18,73 \%$ & FCA & Ovissipour et al., 2014 \\
Lubina asiática (l) & $25 \%$ & Supervivencia, GP, TCE & Srichanun et al., 2014 \\
Lubina europea (a) & $5 \%$ & Peso final, TCE, FCA & Leduc et al., 2018 \\
Lubina europea (l) & $10 \%$ & Supervivencia, final & Kotzamanis et al., 2007 \\
Lubina europea (j) & $3 \%$ & Peso final & Costa et al., 2020 \\
Lubina japonesa (j) & $15 \%$ & Peso final, TCE & Liang et al., 2006 \\
Mero (j) & $15 \%$ & GP, FCA & Mamauag et al., 2016 \\
Pacu (l) & $19,50 \%$ & Peso final & Macedo-Viegas et al., 2004 \\
Pez blanco (j) & $15 \%$ & GP & Ospina-Salazar et al., 2016 \\
Pez gato (a) & $10 \%$ & GP, TCE, FCA, TEP & Rathore et al., 2018 \\
Salmón del Atlántico (j) & $10 \%$ & TCE, FCA, TEP & Egerton et al., 2020 \\
Salmón del Atlántico (ps) & $15 \%$ & TCE & Refstie et al., 2004 \\
Tilapia del Nilo (l) & $4 \%$ & Supervivencia, talla final, PF & Sary et al., 2017 \\
Tilapia del Nilo (pl) & $4,75 \%$ & GP & Da Silva et al.,, 2017 \\
Turbot (j) & $5,4 \%$ & TCE & Wei et al., 2016 \\
Turbot (j) & $3,1-5,4 \%$ & Supervivencia, TCE, FCA & Wei et al., 2019 \\
\hline
\end{tabular}


Tabla 2

Efectos positivos de HPP en el cultivo de crustáceos. Estadios: post-larva (pl), juvenil (j), adulto (a). Indice productivo: TCE (tasa de crecimiento específico), FCA (factor de conversión alimenticia), TEP (tasa de eficiencia proteica) (Adaptado de Martínez-Alvarez et al., 2015)

\begin{tabular}{lrll}
\hline Especie & Dosis HPP & Índice afectado & Referencia \\
\hline Langostino blanco (pl) & $6 \%$ & Peso final & Quinto et al., 2018 \\
Langostino blanco (pl) & $1,52-1,65 \%$ & Peso final, ganancia de peso, TEP & Valle et al., 2015 \\
Langostino blanco (pl) & $1,0-1,5 \%$ & Supervivencia, peso final, FCA & Li et al., 2018 \\
Langostino blanco (j) & $4,4 \%$ & TCE, FCA, TEP & Grey et al., 2009 \\
Langostino blanco (j) & $5 \%$ & Consumo de alimento & Wei et al., 2016 \\
Langostino blanco (j) & $9 \%$ & Peso final, FCA & Córdova y García, 2002 \\
Langostino blanco (a) & $15,2-15,4 \%$ & Supervivencia, peso final, FCA, TEP & Hernández et al., 2011 \\
\hline
\end{tabular}

Sobre la mejora de la tasa supervivencia, dietas suplementadas con HPP presentaron altos performances en langostinos (Hernández et al., 2011; Li et al., 2018). Es poco conocido el accionar de los HPP sobre la respuesta inmune en los crustáceos, sin embargo, niveles de HPP en las dietas permiten una mejora de la respuesta inmune innata y la actividad antioxidante, reflejada en la activación del sistema Profenoloxidasa (sistema proPO), considerado como el sistema inmune más importante en crustáceos (Li et al., 2018).

De acuerdo con los resultados obtenidos por diversos autores, los camarones blancos necesitan niveles moderados de inclusión de HPP para promover su crecimiento, tal como se muestra en la Tabla 2. Estos niveles se deberían probablemente a la absorción prematura por ciertos aminoácidos libres en el tracto gastrointestinal, cambiando la tasa de absorción en desmedro de los aminoácidos en cadenas polipeptídicas, según su fase o estadio de desarrollo.

\subsection{Efectos sobre el desempeño productivo en moluscos}

La acuicultura de moluscos generalmente está enfocada en el cultivo de organismos filtradores como ostras, mejillones, almejas y vieiras. Sin embargo, algunos organismos, como el caso de los abalones y cefalópodos requieren de dietas balanceadas y mixtas para su cultivo.

En abalón Haliotis midae, se estudiaron los efectos de la inclusión de un hidrolizado comercial de pescado, encontrándose una mejora en la ganancia diaria de peso y en la conversión alimenticia (11\% y $14 \%$, respectivamente), en comparación con la dieta control (Goosen et al., 2014). Asimismo, se observó una mejora de la función inmune celular, expresada en el aumento de la actividad fagocítica (Goosen et al., 2014).

Trabajos realizados con cefalópodos han reportado poca aceptación de estos animales hacia las dietas artificiales, dificultando su performance en condiciones de cautiverio. Sin embargo, la inclusión de 5\% de HPP permitió una mejor receptibilidad hacia las dietas suministradas en juveniles de pulpo Octopus maya (Domingues et al., 2007; Rosas et al., 2007). Efectos positivos en la tasa de crecimiento especifico $(0,74 \% /$ día), estimulación de enzimas digestivas y reducción de costos energéticos relacionados con la digestión, se presentaron en $O$. maya, cuando fueron alimentados con dietas conteniendo 15\% de HPP (Aguila et al., 2007). Para el caso de juveniles de 0 . vulgaris, dietas con $10 \mathrm{y}$ $20 \%$ de hidrolizado permitieron un crecimiento de 0,80 y $0,89 \%$ /día, respectivamente (GarcíaGarrido et al., 2007).

$\mathrm{Si}$ bien es cierto, los valores relacionados al crecimiento fueron bajos comparados con las dietas controles, a base de proteínas nativas (alimentos a base cangrejo y calamar), la inclusión de hidrolizados ayudó a una mayor producción de biomasa de los ejemplares, comparada con las dietas experimentales realizados en otros estudios, sugiriéndose que podrían ser una fuente nutricional para hacer factibles las dietas artificiales (Aguila et al., 2017).

El efecto de los alimentos suplementados con HPP sobre el crecimiento de los moluscos no está bien descrito, y si este insumo impacta en el crecimiento de manera positiva o negativa.

\subsection{Efectos sobre el desempeño productivo en algas}

No se encuentran estudios sobre los beneficios de los HPP para este grupo de organismos, salvo la experiencia reportada para Kappaphycus alvarezii, fertilizada con hidrolizado de tilapia $\left(10 \mathrm{~mL} \mathrm{~L}^{-1}\right)$, que presentó un significativo incremento en ganancia de peso y tasa diaria de crecimiento, después de 4 semanas de cultivo. Este estudio demostraría que los HPP podrían ser usados como fertilizantes líquidos para mejorar la producción de macroalgas (Casaclang et al., 2017).

\section{CONCLUSIONES}

Los HPP promueven el crecimiento de varias especies de organismos acuáticos, debido a sus características aromatizantes, perfil balanceado de aminoácidos y péptidos de bajo peso molecular, alta digestibilidad que permite una rápida absorción intestinal y aprovechamiento, pudiéndose constituir como una opción recomendable en la alimentación acuícola. Por otro lado, se estaría dando un uso a los residuos, desperdicios o materiales que suelen ser descartados por la industria pesquera y acuícola, aportando a la "economía circular". Sin embargo, son necesarios estudios que permitan definir el nivel de inclusión óptimo para la especie objetivo, según estadio de desarrollo, condiciones de cultivo, área geográfica, entre otros, y alcanzar la eficiencia productiva. 


\section{AGRADECIMIENTOS}

Al profesor Javier Coasaca Céspedes por sus contribuciones para el mejor desarrollo del trabajo, así como a los integrantes del Grupo de Estudios en
Acuicultura Sostenible (GEAS), por sus valiosos aportes en el campo del conocimiento de la acuicultura.

\section{REFERENCIAS BIBLIOGRÁFICAS}

Aguila, J., Cuzon, G., Pascual, C., Domingues, P. M., Gaxiola, G., Sánchez, A., Maldonado, T., \& Rosas, C. (2007). The effects of fish hydrolysate (CPSP) level on Octopus maya (Voss and Solis) diet: Digestive enzyme activity, blood metabolites, and energy balance. Aquaculture, 273(4), 641-655.

Akness, A., Hope, B., Høstmark, Ø., \& Albrektsen, S. (2006a). Inclusion of size fractionated fish hydrolysate in high plant protein diets for Atlantic cod, Gadus morhua. Aquaculture, 261(3), 1102-1110.

Akness, A., Hope, B., Jönsson, E., Björnsson, B. T., \& Albrektsen, S. (2006b). Size-fractionated fish hydrolysate as feed ingredient for rainbow trout (Oncorhynchus mykiss) fed high plant protein diets. I: Growth, growth regulation and feed utilization. Aquaculture, 261(1), 305-317.

Bui, H. T. D., Khosravi, S., Fournier, V., Herault, M., \& Lee, K.-J. (2014). Growth performance, feed utilization, innate immunity, digestibility and disease resistance of juvenile red seabream (Pagrus major) fed diets supplemented with protein hydrolysates. Aquaculture, 418-419, 11-16.

Cai, Z., Li, W., Mai, K., Xu, W., Zhang, Y., \& Ai, Q. (2015). Effects of dietary size-fractionated fish hydrolysates on growth, activities of digestive enzymes and aminotransferases and expression of some protein metabolism related genes in large yellow croaker (Larimichthys crocea) larvae. Aquaculture, 440, 40-47.

Casaclang, J. R., Gregorio, A. C., Resuello, R. B., Reyes, C. J. A., Bernardo-Fernandez, J., \& Gonzales-Plasus, M. M. (2017). Fish hydrolysate derived from fish waste increased the growth of Kappaphycus alvarezii, 10(5), 1150-1156.

Chalamaiah, M., Dinesh, B., Hemalatha, R., \& Jyothirmayi, T. (2012). Fish protein hydrolysates: Proximate composition, amino acid composition, antioxidant activities and applications: A review. Food Chemistry, 135(4), 3020-3038.

Chotikachinda, R., Tantikitti, C., Benjakul, S., Rustad, T., \& Kumarnsit, E. (2013). Production of protein hydrolysates from skipjack tuna (Katsuwonus pelamis) viscera as feeding attractants for Asian seabass (Lates calcarifer). Aquaculture Nutrition, 19(5), 773-784.

Córdova-Murueta, J. H., \& García-Carreño, F. L. (2002). Nutritive value of squid and hydrolyzed protein supplement in shrimp feed. Aquaculture, 210(1), 371-384.

Costa, M., Costas, B., Machado, M., Teixeira, C., Fernández-Boo, S., Sá, T., Batista, S., Marques, A., Miranda, F., \& Valente, L. M. P. (2020). Anchovy and giant squid hydrolysates can enhance growth and the immune response of European seabass (Dicentrarchus labrax) fed plant-protein-based diets. Aquaculture, 523, 735182.

Domingues, P. M., López, N., Muñoz, J. A., Maldonado, T., Gaxiola, G., \& Rosas, C. (2007). Effects of a dry pelleted diet on growth and survival of the Yucatan octopus, Octopus maya. Aquaculture Nutrition, 13(4), 273-280.

Egerton, S., Wan, A., Murphy, K., Collins, F., Ahern, G., Sugrue, I., Busca, K., Egan, F., Muller, N., Whooley, J., McGinnity, P., Culloty, S., Ross, R. P., \& Stanton, C. (2020). Replacing fishmeal with plant protein in Atlantic salmon (Salmo salar) diets by supplementation with fish protein hydrolysate. Scientific Reports, 10(1), 4194.

FAO. (2020). El estado mundial de la pesca y la acuicultura 2020. La sostenibilidad en acción. FAO.

Galla, N. R., Pamidighantam, P. R., Akula, S., \& Karakala, B. (2012). Functional properties and in vitro antioxidant activity of roe protein hydrolysates of Channa striatus and Labeo rohita. Food Chemistry, 135(3), 1479-1484.

Goosen, N. J., de Wet, L. F., \& Görgens, J. F. (2014). The effects of protein hydrolysates on the immunity and growth of the abalone Haliotis midae. Aquaculture, 428-429, 243-248.

Grey, M., Forster, I., Dominy, W., Ako, H., \& Giesen, A. F. (2009). Validation of a Feeding Stimulant Bioassay Using Fish Hydrolysates for the Pacific White Shrimp, Litopenaeus vannamei. J. of the World Aquaculture Society, 40(4), 547-555.

Ha, N., Jesus, G. F. A., Gonçalves, A. F. N., de Oliveira, N. S., Sugai, J. K., Pessatti, M. L., Mouriño, J. L. P., \& El Hadi Perez Fabregat, T. (2019). Sardine (Sardinella spp.) protein hydrolysate as growth promoter in South American catfish (Rhamdia quelen) feeding: Productive performance, digestive enzymes activity, morphometry and intestinal microbiology. Aquaculture, 500, 99-106.

Halim, N. R. A., Yusof, H. M., \& Sarbon, N. M. (2016). Functional and bioactive properties of fish protein hydolysates and peptides: A comprehensive review. Trends in Food Science \& Technology, 51, 24-33.

Han, D., Shan, X., Zhang, W., Chen, Y., Wang, Q., Li, Z., Zhang, G., Xu, P., Li, J., Xie, S., Mai, K., Tang, Q., \& Silva, S. S. D. (2018). A revisit to fishmeal usage and associated consequences in Chinese aquaculture. Reviews in Aquaculture, 10(2), 493-507.

He, S., Franco, C., \& Zhang, W. (2013). Functions, applications and production of protein hydrolysates from fish processing coproducts. Food Research International, 50(1), 289-297.

Hernández, C., Olvera-Novoa, M. A., Smith, D. M., Hardy, R. W., \& Gonzalez-Rodriguez, B. (2011). Enhancement of shrimp Litopenaeus vannamei diets based on terrestrial protein sources via the inclusion of tuna by-product protein hydrolysates. Aquaculture, 317(1), 117-123.

Ho, T. C. W., Li-Chan, E. C. Y., Skura, B. J., Higgs, D. A., \& Dosanjh, B. (2014). Pacific hake (Merluccius productus Ayres, 1855) hydrolysates as feed attractants for juvenile Chinook salmon (Oncorhynchus tshawytscha Walbaum, 1792). Aquaculture Research, 45(7), 1140-1152.

Khosravi, S., Rahimnejad, S., Herault, M., Fournier, V., Lee, C.-R., Dio Bui, H. T., Jeong, J.-B., \& Lee, K.-J. (2015). Effects of protein hydrolysates supplementation in low fish meal diets on growth performance, innate immunity and disease resistance of red sea bream Pagrus major. Fish \& Shellfish Immunology, 45(2), 858-868.

Klompong, V., Benjakul, S., Kantachote, D., \& Shahidi, F. (2009). Characteristics and Use of Yellow Stripe Trevally Hydrolysate as Culture Media. Journal of Food Science, 74(6), S219-S225.

Kotzamanis, Y. P., Gisbert, E., Gatesoupe, F. J., Zambonino Infante, J., \& Cahu, C. (2007). Effects of different dietary levels of fish protein hydrolysates on growth, digestive enzymes, gut microbiota, and resistance to Vibrio anguillarum in European sea bass (Dicentrarchus labrax) larvae. Comparative Biochemistry and Physiology Part A: Molecular \& Integrative Physiology, 147(1), 205-214.

Kvåle, A., Harboe, T., Mangor-Jensen, A., \& Hamre, K. (2009). Effects of protein hydrolysate in weaning diets for Atlantic cod (Gadus morhua L.) and Atlantic halibut (Hippoglossus hippoglossus L.). Aquaculture Nutrition, 15(2), 218-227.

Leduc, A., Zatylny-Gaudin, C., Robert, M., Corre, E., Corguille, G. L., Castel, H., Lefevre-Scelles, A., Fournier, V., Gisbert, E., Andree, K. B., \& Henry, J. (2018). Dietary aquaculture by-product hydrolysates: Impact on the transcriptomic response of the intestinal mucosa of European seabass (Dicentrarchus labrax) fed low fish meal diets. BMC Genomics, 19(1), 396.

Li, X., Wang, L., Zhang, C., Rahimnejad, S., Song, K., \& Yuan, X. (2018). Effects of Supplementing Low-Molecular-Weight Fish Hydrolysate in High Soybean Meal Diets on Growth, Antioxidant Activity and Non-Specific Immune Response of Pacific White Shrimp (Litopenaeus vannamei). Turkish Journal of Fisheries and Aquatic Sciences, 18(5), 717-727.

Liang, M., Wang, J., Chang, Q., \& Mai, K. (2006). Effects of different levels of fish protein hydrolysate in the diet on the nonspecific immunity of Japanese sea bass, Lateolabrax japonicus (Cuvieret Valenciennes, 1828). Aquaculture Research, 37(1), 102-106.

Macedo-Viegas, E. M., Portella, M. C., \& Carneiro, D. J. (2004). Utilization of Fish Protein Hydrolysate in Prepared Diets for Pacu, Piaractus mesopotamicus, Larvae. Journal of Applied Aquaculture, 14(3-4), 101-112.

Mamauag, R. E. P., \& Ragaza, J. A. (2016). Growth and feed performance, digestibility and acute stress response of juvenile grouper (Epinephelus fuscoguttatus) fed diets with hydrolysate from milkfish offal. Aquaculture Research, 48(4), 1638-1647.

Martínez-Alvarez, O., Chamorro, S., \& Brenes, A. (2015). Protein hydrolysates from animal processing by-products as a source 
of bioactive molecules with interest in animal feeding: A review. Food Research International, 73, 204-212.

Masuda, Y., Jinbo, T., Imaizumi, H., Furuita, H., Matsunari, H., Murashita, K., Fujimoto, H., Nagao, J., \& Kawakami, Y. (2013). A step forward in development of fish protein hydrolysatebased diets for larvae of Japanese eel Anguilla japonica. Fisheries Science, 79(4), 681-688.

Nguyen, H. T. M., Pérez-Gálvez, R., \& Bergé, J. P. (2012). Effect of diets containing tuna head hydrolysates on the survival and growth of shrimp Penaeus vannamei. Aquaculture, 324-325, 127-134.

Ospina-Salazar, G. H., Ríos-Durán, M. G., Toledo-Cuevas, E. M., \& Martínez-Palacios, C. A. (2016). The effects of fish hydrolysate and soy protein isolate on the growth performance, body composition and digestibility of juvenile pike silverside, Chirostoma estor. Animal Feed Science and Technology, 220, 168-179.

Ovissipour, M., Kenari, A. A., Nazari, R., Motamedzadegan, A., \& Rasco, B. (2014). Tuna viscera protein hydrolysate: Nutritive and disease resistance properties for Persian sturgeon (Acipenser persicus L.) larvae. Aquaculture Research, 45(4), 591-601.

Quinto, B. P. T., Albuquerque, J. V., Bezerra, R. S., Peixoto, S., \& Soares, R. (2018). Replacement of fishmeal by two types of fish protein hydrolysate in feed for postlarval shrimp Litopenaeus vannamei. Aquaculture Nutrition, 24(2), 768776.

Rathore, S. S., Chandravanshi, A., Chandravanshi, P., Srinivasa, K. H., Rakesh, K., Mamun, M. A. A., \& Nasren, S. (2018). Optimization of Fish Hydrolysate Preparation and its Effect on Growth and Feed Utilization of Magur (Clarias batrachus). Bull. Env. Pharmacol. Life Sci., 7(11), 78-83.

Refstie, S., Olli, J. J., \& Standal, H. (2004). Feed intake, growth, and protein utilisation by post-smolt Atlantic salmon (Salmo salar) in response to graded levels of fish protein hydrolysate in the diet. Aquaculture, 239(1), 331-349.

Rosas, C., Cuzon, G., Pascual, C., Gaxiola, G., Chay, D., López, N., Maldonado, T., \& Domingues, P. M. (2007). Energy balance of Octopus maya fed crab or an artificial diet. Marine Biology, 152(2), 371-381.

Sary, C., Paris, L. D. de, Bernardi, D. M., Lewandowiski, V., Signor, A., \& Boscolo, W. R. (2017). Tilapia by-product hydrolysate powder in diets for Nile tilapia larvae. Acta Scientiarum. Animal Sciences, 39(1), 1-6.

Sheriff, S. A., Sundaram, B., Ramamoorthy, B., \& Ponnusamy, P. (2014). Synthesis and in vitro antioxidant functions of protein hydrolysate from backbones of Rastrelliger kanagurta by proteolytic enzymes. Saudi Journal of Biological Sciences, 21(1), 19-26.

Siddik, M. A. B., Howieson, J., Fotedar, R., \& Partridge, G. J. (2021). Enzymatic fish protein hydrolysates in finfish aquaculture: A review. Reviews in Aquaculture, 13(1), 406-430.

Silva, T. C. da, Rocha, J. D. M., Moreira, P., Signor, A., Boscolo, W. R., Silva, T. C. da, Rocha, J. D. M., Moreira, P., Signor, A., \& Boscolo, W. R. (2017). Fish protein hydrolysate in diets for Nile tilapia post-larvae. Pesquisa Agropecuária Brasileira, 52(7), 485-492.

Silva, J. F. X., Ribeiro, K., Silva, J. F., Cahú, T. B., \& Bezerra, R. S. (2014). Utilization of tilapia processing waste for the production of fish protein hydrolysate. Animal Feed Science and Technology, 196, 96-106.

Srichanun, M., Tantikitti, C., Kortner, T. M., Krogdahl, Å., \& Chotikachinda, R. (2014). Effects of different protein hydrolysate products and levels on growth, survival rate and digestive capacity in Asian seabass (Lates calcarifer Bloch) larvae. Aquaculture, 428-429, 195-202.

Tang, H., Wu, T., Zhao, Z., \& Pan, X. (2008). Effects of fish protein hydrolysate on growth performance and humoral immune response in large yellow croaker (Pseudosciaena crocea R.). Journal of Zhejiang University SCIENCE B, 9(9), 684-690.

Valle, B. C. S., Dantas, E. M., Silva, J. F. X., Bezerra, R. S., Correia, E. S., Peixoto, S. R. M., \& Soares, R. B. (2015). Replacement of fishmeal by fish protein hydrolysate and biofloc in the diets of Litopenaeus vannamei postlarvae. Aquaculture Nutrition, 21(1), 105-112.

Villamil, O., Váquiro, H., \& Solanilla, J. F. (2017). Fish viscera protein hydrolysates: Production, potential applications and functional and bioactive properties. Food Chemistry, 224, 160-171.

Wei, Y., Liang, M., Mu, Y., Zheng, K., \& Xu, H. (2016). The effect of ultrafiltered fish protein hydrolysate level on growth performance, protein digestibility and mRNA expression of PepT1 in juvenile turbot (Scophthalmus maximus L.). Aquaculture Nutrition, 22(5), 1006-1017.

Wei, Yuliang, Liang, M., \& Xu, H. (2019). Fish protein hydrolysate affected amino acid absorption and related gene expressions of IGF-1/AKT pathways in turbot (Scophthalmus maximus). Aquaculture Nutrition, 26(1), 145-155.

Wisuthiphaet, N., Kongruang, S., \& Chamcheun, C. (2015). Production of Fish Protein Hydrolysates by Acid and Enzymatic Hydrolysis. Journal of Medical and Bioengineering, 4(6), 466-470.

Wosniak, B., Hessa Melim, E. W., Ha, N., Uczay, J., Pilatti, C., Pessatti, M. L., \& Perez Fabregat, T. E. H. (2016). Effect of diets containing different types of sardine waste (Sardinella sp.) protein hydrolysate on the performance and intestinal morphometry of silver catfish juveniles (Rhamdia quelen). Latin American Journal of Aquatic Research, 44(5), 957-966.

$\mathrm{Xu}, \mathrm{H} ., \mathrm{Mu}, \mathrm{Y}$., Zhang, Y., Li, J., Liang, M., Zheng, K., \& Wei, Y. (2016). Graded levels of fish protein hydrolysate in high plant diets for turbot (Scophthalmus maximus): Effects on growth performance and lipid accumulation. Aquaculture, 454, 140147.

Zamora-Sillero, J., Gharsallaoui, A., \& Prentice, C. (2018). Peptides from Fish By-product Protein Hydrolysates and Its Functional Properties: An Overview. Marine Biotechnology, 20(2), 118-130.

Zheng, K., Liang, M., Yao, H., Wang, J., \& Chang, Q. (2012). Effect of dietary fish protein hydrolysate on growth, feed utilization and IGF-I levels of Japanese flounder (Paralichthys olivaceus). Aquaculture Nutrition, 18(3), 297-303.

Zheng, K., Liang M., Yao H., Wang J., \& Chang Qing. (2013). Effect of size-fractionated fish protein hydrolysate on growth and feed utilization of turbot (Scophthalmus maximus L.). Aquaculture Research, 44(6), 895-902. 